Rev. Elev. Méd. vét. Pays trop., 1977, 30 (2) : 135-139.

\title{
Note sur les conséquences pratiques de la lyophilisation des spores de Bacillus anthracis
}

\author{
par P. C. LEFEVRE $\left(^{*}\right)$ \\ (avec la collaboration technique de M. MOYALBAYE)
}

\begin{abstract}
RÉSUMÉ
Des essais de lyophilisation des spores de Bacillus anthracis (souche STERNE) ont montré que le pourcentage de spores revivifiables dépend des milieux de lyophilisation utilisés : le lait écrémé et le milieu de Weybridge sont d'excellents protecteurs contrairement au milieu à la néopeptone.

En outre, les spores lyophilisées sont «fragilisées» et le pourcentage de spores après reconstitution dépend du diluant: le sérum physiologique n'a aucune action nocive à l'inverse de la solution molaire de sulfate de magnésium qui détruit 50 p. 100 des spores.
\end{abstract}

\section{INTRODUCTION}

Dans les pays où l'élevage nomade domine, les vaccins mixtes lyophilisés sont particulièrement recherchés $(6,9,10)$, en raison de leur meilleure conservation et de leur facilité d'utilisation sur le terrain.

Dans le but de mettre au point soit un vaccin monovalent lyophilisé (4), soit un vaccin mixte péripneumonie-charbon bactéridien, une série d'expériences a été menée pour préciser l'influence de la lyophilisation sur les spores de Bacillus anthracis souche Sterne.

En effet, plusieurs auteurs ont déjà souligné le caractère néfaste de celle-là sur celles-ci $(1,2,10)$. Pour PROVOST et collab. 60 p. 100 des spores sont détruites lors de la lyophilisatıon. Toutefois des antibiotiques ayant été incorporés dans le vaccin mixte (du fait du milieu de croissance de Mycoplasma mycoides) et bien que les diverses opérations aient été menées sous froid, il est impossible de dire avec précision quelle

(*) I. E. M. V. T., Laboratoire de Farcha, B. P. 433, N'Djamena (Tchad). est la part de la lyophilisation dans cette perte de spores.

De plus, toujours selon ces mêmes auteurs, les spores lyophilisées seraient sensibles à l'action du sulfate de magnésium. Cette observation surtout est très importante car la solution molaire de $\mathrm{Mg} \mathrm{SO}_{4}$ est très largement utilisée dans les pays d'Afrique Centrale pour la reconstitution des vaccins lyophilisés en raison de son action thermo-protectrice pour Mycoplasma mycoides et le virus bovipestique $(7,12)$.

De même, selon les auteurs roumains (ALBOIU et collab.) les spores sont «fragilisées » et perdent une grande partie de leur pouvoir de résistance à la chaleur, la lumière et les antiseptiques mais ils ne précisent pas l'ordre de grandeur de la perte en spores revivifiables. En revanche, RAMYAR et BAHARSEFAT ne signalent pas ce problème dans la préparation de leur vaccin mixte lyophilisé charbon bactéridien-clavelée.

Aussi, avant d'introduire la valence charbon bactéridien d'une importance capitale dans les pays du Sahel (13) dans un vaccin mixte lyophilisé, il nous était nécessaire de déterminer avec exactitude le pourcentage de spores revivi- 
fiables après lyophilisation et ceci en faisant varier les milieux protecteurs et les diluants de reconstitution. Par ailleurs, l'adjonction de saponine dans le vaccin anti-charbon bactéridien étant nécessaire, une étude portant sur la préparation de ce vaccin saponiné lyophilisé a été entreprise séparément.

\section{MATÉRIEL ET MÉTHODE}

\section{MATÉRIEL}

1) Toutes les expériences ont été conduites avec la couche STERNE 34 F2 du Laboratoire International des étalons biologiques, Weybridge (Angleterre).

2) Quatre milieux de lyophilisation ont été employés :

- lait écrémé ;

- milieu de Weybridge (3): hydrolysat de caséine $=25 \mathrm{~g}$; saccharose $=5 \mathrm{~g}$; glutamate de $\operatorname{sodium}=5 \mathrm{~g}$; eau distillée $=11$;

- milieu de Wiktor (5): phosphate monopotassique $=2,7 \mathrm{~g}$; peptone $=20 \mathrm{~g}$; lactose $=20 \mathrm{~g}$; eau distillée $=11$;

- milieu à la néopeptone: néopeptone (DIFCO) $=110 \mathrm{~g}$; eau distillée $=11$.

Les trois premiers milieux ont été stérilisés par filtration sur EKS II après ajustement du $\mathrm{pH}$ à 7,2-7,4. Le lait écrémé a été stérilisé à $120^{\circ}$ pendant $30 \mathrm{mn}$.

3) La lyophilisation a été menée sur un appareil Major (FROILABO-SOGEV). On a, dans un premier temps, réalisé une lyophilisation primaire de -60 à $-25^{\circ} \mathrm{C}$ en $24 \mathrm{~h}$, puis une dessiccation secondaire de -25 à $+20^{\circ} \mathrm{C}$ pendant une vingtaine d'heures. Les flacons ont été bouchés sous atmosphère d'azote.

4) On a utilisé comme diluant de reconstitution soit du sérum physiologique, soit une solution molaire de sulfate de magnésium.

\section{MÉTHODE}

1) La technique de culture de Bacillus anthracis est la technique classique de production du vaccin anticharbonneux sporulé selon les normes internationales de l'O. M. S. (4). La récolte est effectuée avec un minimum d'eau distillée et la suspension obtenue est ensuite chauffée $1 \mathrm{~h}$ à $65^{\circ} \mathrm{C}$ pour détruire les corps microbiens tout en conservant les spores. Après chauffage, la suspension est mise sur agitateur magnétique. Quand la répartition des spores est homogène, 4 fractions aliquotes sont mélangées à parties égales avec les milieux de lyophilisation. Les mélanges sont ensuite répartis dans des flacons de $20 \mathrm{ml}$ à raison de $5 \mathrm{ml}$ par flacon et portés dans le lyophilisateur. La lyophilisation terminée, les flacons sont stockés à $-20^{\circ},+4^{\circ}$ et $37^{\circ} \mathrm{C}$. Les titrages sont effectués immédiatement après, puis à 1,2 et 3 mois en utilisant les 2 diluants de reconstitution.

2) Parallèlement et pour savoir si l'action délétère du sulfate de magnésium s'exerce sur les spores en général ou seulement sur des spores lyophilisées et peut-être « fragilisées ", des spores fraîchement récoltées sont immédiatement mélangées avec du sérum physiologique ou une solution molaire de sulfate de magnésium dans des proportions équivalentes. Les suspensions obtenues sont mises sur agitateur 1 h 30 avant titrage. Cette expérience a été répétée 4 fois avec des suspensions différentes.

3) Lors du titrage, les flacons lyophilisés sont repris dans $20 \mathrm{ml}$ de diluant (sérum physiologique ou solution molaire de sulfate de magnésium) et mis en agitation pendant $1 \mathrm{~h} 30$. Le volume de $20 \mathrm{ml}$ et le temps de $1 \mathrm{~h} 30$ ont été choisis pour que l'action du sulfate de magnésium soit maximale. Les titrages sont effectués par comptage des colonies en boîte de Petri après dilutions décimales ( 5 boîtes par dilution). Les volumes étant différents $-5 \mathrm{ml}$ avant lyophilisation et $20 \mathrm{ml}$ après - les titres sont exprimés en spores par flacon.

\section{RÉSULTATS}

Les résultats sont colligés dans les tableaux I, II et III.

\section{DISCUSSION. CONCLUSION}

$\mathrm{Au}$ vu des tableaux suivants, il est possible de faire un certain nombre de constatations:

1) Les milieux de lyophilisation (tabl. I).

Le lait écrémé et le milieu de Weybridge se révèlent d'excellents protecteurs pour les spores 
TABL. N*I-Titre des spores de Saci: Jus anthravio avant et après lyophilisation dans divers milieux protecteurs.

\begin{tabular}{|c|c|c|c|}
\hline & \multirow[b]{2}{*}{$\begin{array}{l}\text { Titre avant } \\
\text { lyophilisatzon (1) }\end{array}$} & \multicolumn{2}{|c|}{ 'Titre après lyophlisation } \\
\hline & & $\begin{array}{c}\text { Sérum } \\
\text { physiologique }\end{array}$ & $\begin{array}{c}\text { Solution molarre } \\
\text { de } \mathrm{Mg} \mathrm{SO}\end{array}$ \\
\hline $\begin{array}{l}\text { Spores }+ \\
\text { lait êcrémé }\end{array}$ & $\begin{array}{c}1890 \times 10^{6} \\
1740 \quad 2045:\end{array}$ & $\begin{array}{c}1820 \times 10^{6} \\
1680 \quad 1970\end{array}$ & $\begin{array}{c}1087 \times 10^{6} \\
1008 \times 1165\end{array}$ \\
\hline $\begin{array}{l}\text { Spores + } \\
\text { milieu de Weybridge }\end{array}$ & $\begin{array}{cc}1845 \times 10^{6} \\
1695 & 1995\end{array}$ & $\begin{array}{c}1990 \times 10^{6} \\
1838 \quad 2146\end{array}$ & $\begin{array}{c}916 \times 10^{6} \\
850 \quad 980\end{array}$ \\
\hline $\begin{array}{l}\text { Spores + } \\
\text { milieu de Wiktor }\end{array}$ & $\begin{array}{cc}1705 & \times 10^{6} \\
1575 & 1825\end{array}$ & $\begin{array}{c}1476 \times 10^{6} \\
1360 \quad 1600\end{array}$ & $\begin{array}{c}880 \times 10^{6} \\
762 \quad 998\end{array}$ \\
\hline $\begin{array}{l}\text { Spores + } \\
\text { Nêopeptone }\end{array}$ & $\begin{array}{c}1755 \times 10^{6} \\
1625\end{array}$ & $\begin{array}{c}727 \times 10^{6} \\
625 \quad 1890\end{array}$ & $\begin{array}{c}410 \times 10^{6} \\
338 \quad 432\end{array}$ \\
\hline
\end{tabular}

(1) le thtre moyen avant lyophlisation est de $1800 \times 10^{\circ}$ spores avec un intervalle de confiance à 95 p. 100 comprus entre $1655 \times 10^{\circ}$ et $1940 \times 10^{\circ}$ spores par flacon.

- les chiffres indiqués à l'alinéa inférieur sont les limates de l'intervalle de confiance à 85 p. 100.

de Bacillus anthracis. Aucune perte de spores n'est enregistrée après lyophilisation dans ces 2 milieux et reconstitution dans du sérum physiologique.

En revanche, le milieu à la néopeptone et le milieu de Wiktor sont nettement moins favorables, notamment le premier avec lequel on ne retrouve, après lyophilisation, que 40 p. 100 des spores.

\section{2) Les diluants de reconstitution (tabl. II).}

Les observations de PROVOST et collab. sur l'action délétère d'une solution molaire de sulfate de magnésium et celles d'ALBOIU et collab. sur la fragilisation des spores lyophilisées sont toutes les deux amplement vérifiées.

Les spores lyophilisées avec le milieu de Weybridge ou le lait écrémé et reconstituées dans du sérum physiologique ne subissent aucune perte.

En revanche, les mêmes spores reconstituées dans la solution molaire de $\mathrm{MgSO}_{4}$ sont détruites à 50 p. 100 .

Après lyophilisation dans le milieu à la néopeptone et reconstitution dans la solution molaire de $\mathrm{Mg} \mathrm{SO}_{4}, 67$ p. 100 des spores sont détruites. Le diluant de reconstitution est responsable de la perte de près de la moitié des spores non détruites lors de la lyophilisation.

Or, le tableau II montre à l'évidence que cette solution n'a aucune action nocive sur des spores non lyophilisées.

La lyophilisation a donc pour effet d'entraîner une très nette diminution de la résistance
TABL. $N^{\circ}$ II-Action de la solution molaire $\mathrm{Mg} \mathrm{S0} 4$ sur les spores non tyophilisées

\begin{tabular}{|c|c|c|}
\hline $\begin{array}{c}\text { Expérience } \\
\mathrm{n}^{\circ}\end{array}$ & $\begin{array}{c}\text { Sérum } \\
\text { physiologique }\end{array}$ & $\begin{array}{c}\text { Solution molaire } \\
\text { de } \mathrm{Mg} \mathrm{SO}\end{array}$ \\
\hline 1 & $8^{10 \times 10^{6}} 12$ & $7^{9,3 \times 10^{6}} 11$ \\
\hline 2 & $3^{38 \times 10^{6}}$ & $4^{46} \times 10^{6}$ \\
\hline 3 & $92^{93 \times 10^{6}}$ & $4^{84} \times 10^{6}$ \\
\hline 4 & $132 \times 10^{6}$ & $190 \times 10^{6}$ \\
\hline
\end{tabular}

des spores. Cette notion devra être gardée en mémoire car, lors de la production d'un vaccin, il est nécessaire de tenir compte des baisses de titre cumulées depuis la sortie du laboratoire jusqu'au moment de l'injection et dues à de nombreux facteurs (chaleur, exposition à la lumière, etc...).

\section{3) Résistance des spores lyophilisées conservées} à diverses températures (tabl. III).

La résistance des spores lyophilisées à $4^{\circ}$ et $37^{\circ}$ semble satisfaisante : les écarts des titres du vaccin conservé à différentes températures ne sont pas significatifs.

La lyophilisation des spores de Bacillus anthracis n'est pas une opération banale et sans danger pour ces dernières. Le choix du milieu de lyophilisation est très important: le lait écrémé et le milieu de Weybridge sont d'excellents protecteurs alors que le milieu à la néopeptone est à proscrire. De même le diluant de reconstitution n'est pas sans action du fait de la 
TABL. N•III-Rësistance des spores de Bacillus conthracis lyophilisẻes en milieu de Weybridge et conservées à $-20^{\circ},+4^{\circ}$ et $+37^{\circ} \mathrm{C}$.

\begin{tabular}{|c|c|c|c|}
\hline & \multicolumn{3}{|c|}{ Reconstitution dans du sérum physiologique } \\
\hline & $-20^{\circ} \mathrm{C}$ & $+4^{\circ} \mathrm{C}$ & $+37^{\circ} \mathrm{C}$ \\
\hline 1 mois & $\begin{array}{ll}1650 \times & 10^{6} \\
1504 & 1795\end{array}$ & $\begin{array}{ll}1658 \times 10^{6} \\
1566 & 1754\end{array}$ & $\begin{array}{l}1314 \times 10^{6} \\
1234 \times 1324\end{array}$ \\
\hline 2 mois & $\begin{array}{ll}1638 \times 10^{6} \\
1540 \times 1736\end{array}$ & $\begin{array}{ll}1312 \times 10^{6} \\
1188 & 1436\end{array}$ & $\begin{array}{c}1436 \times 10^{6} \\
1304 \times 1568\end{array}$ \\
\hline 3 mois & $\begin{array}{l}1510 \times 10^{6} \\
1424 \\
1594\end{array}$ & $\begin{array}{l}1720 \times 10^{6} \\
1630 \times 1808\end{array}$ & $\begin{array}{l}1340 \times 10^{6} \\
1258 \times 1422\end{array}$ \\
\hline
\end{tabular}

«fragilisation » des spores lyophilisées. Dans les pays où la solution molaire de sulfate de magnésium est utilisée comme diluant, les titres en spores charbonneuses devront être doublés lors de la préparation du vaccin pour que, une heure et demi après reconstitution, le titre de $5 \times 10^{6}$ par dose vaccinale soit garanti.

Cette contrainte, si elle augmente sensiblement le prix de revient d'un tel vaccin, offre par contre la certitude de résultats vaccinaux satis- faisants, à la suite de la seule et unique intervention qui est de règle dans les conditions de la prophylaxie médicale en région d'élevage extensif.

\section{REMERCIEMENTS}

Nous remercions le Directeur du Laboratoire de Farcha, le Docteur A. PROVOST, pour nous avoir permis de mener à bien ce travail.

\section{SUMMARY}

\section{Practical consequences of freeze drying} of Bacillus anthracis spores

Freeze dry1ng trials of Bacillus anthracis spores (STERNE'S strain) showed that the percentage of living spores after lyophilization depends on the freeze drying medium : skim milk and the Weybridge medium give a better protection than the neopeptone medium.

The lyophilized spores have a decreased resistance and the percentage of living spores after reconstitution depends on the rehydrating solution: physiologic saline has no harmful effect in opposition with the magnesium sulfate solution which destroys 50 per cent of the spores.

\section{RESUMEN}

\section{Consecuencias prácticas de la liofilización} de las esporas de Bacillus anthracis

Ensayos de liofilización de las esporas de Bacillus anthracis (cepa de STERNE) mostrarón que el porcentage de esporas viables depiende de los medios utilizados: la leche desnatada y el medio de Weybridge se revelan buenos protectores en contra al medio con neopeptone.

Las esporas liofilizadas no son tanto resistentes y el porcentaje de esporas después de la reconstitución depiende del diluente : el suero fisiologico no está nocivo pero una solución de sulfato de magnesio destruye 50 por ciento de las esporas.

\section{BIBLIOGRAPHIE}

1. ALBOIU (M.), SERBAN (M.), PRODAN (D.). Est-ce qu'on peut créer des souches bactériennes résistantes à l'action destructrice de la lyophilisation? (en roumain). Lucr. Inst. Cerc. vet. Biopr. Pasteur, 1969/1970, $7: 231-241$.
2. ALBOIU (M.), SERBAN (M.), PRODAN (D.). Influence de la lyophilisation sur la résistance des spores de Bacillus anthracis (en roumain). Lucr. Inst. Cerc. vet. Biopr. Pasteur, 1970, 8 : 189-206. 
3. BOYCE (K. J.), EDGAR (A. W.). Production of freeze dried Brucella abortus strain 19 vaccine. J. appl. Bact., 1966, 29 : 401-408.

4. O. M. S. Normes relatives au vaccin anticharbonneux sporulé (vivant, à usage vétérinaire) $19^{\epsilon}$ rapport des experts de la stardardisation biologique, Genève, O. M. S., 1967 (rapport n 361 ).

5. O. M. S. La rage : technique de laboratoire. Genève, O. M. S., $1967, \mathrm{n}^{\circ} 23$.

6. PROVOST (A.). Principe de production d'un vaccin mixte associé antibovipestique antipérıpneumonie inoculé en un seul temps. Bull. epizoot. Dis. Afr., $1969,17: 7-10$.

7. PROVOST (A.). Activité thermoprotectrice de la solution molaire de sulfate de magnésium sur l'inactivation thermique de Mycoplasma mycoides en phase liquide. C. R. Acad. Sci. Paris, 1970, $270 \mathrm{D}: 3156-3157$.

8. PROVOST (A.). Résultats et perspectives de l'application de vaccins nouveaux dans la péripneumonie. $I^{\mathrm{e}}$ conf. int. Inst. Méd. vét. trop., Berlin, 4-7 octobre 1976.
9. PROVOST (A.), BORREDON (C.). Un vaccin mixte antibovipestique-antipéripneumonique lyophilisé utilisable sur le terrain sans réfrigération. Rev. Elev. Méd. vét. Pays trop., 1974, 27 (3) : 251-263.

10. PROVOST (A.), BORREDON (C.), BOCQUET (P.). Un vaccin mixte trivalent contre la peste bovine, la péripneumonie et le charbon bactéridien. Rev. Elev. Méd. vét. Pays trop., 1974, 27 (4) : 385-395.

11. RAMYAR (H.), BAHARSEFAT (M.). A new approach to active immunization of sheep by a combined sheep-pox and anthrax vaccine. Arch. Inst. Razi, 1970, 22 : 123-128.

12. ROBIN (P.), BOURDIN (P.). Note sur l'action du sulfate de sodium, du sulfate de magnésium et du chlorure de magnésium sur le virus de la peste bovine adapté aux cultures cellulaires. Rev. Elev. Méd. vét. Pays trop., 1966, 19 : 451-456.

13. SIROL (J.), DELPY (P.), GUARD (O.). Une épidémie de charbon humain : à propos de 25 cas observés à 1'hôpital de Fort-Lamy, Tchad. Presse Méd., 1971, 79 :1635-1638.

14. STERNE (M.). The use of anthrax vaccine prepared from avirulent (uncapsulated) variants of Bacillus anthracis. Onderstepoort J. vet. Sci., 1939, 13: 307-312. 